\title{
Chemical Evolution of the Small Magellanic Cloud: The Planetary Nebulae
}

\author{
Thais P. Idiart \& Roberto D.D. Costa \\ IAG/USP-Departamento de Astronomia; Av. Miguel Stéfano 4200; \\ 04301-904 - São Paulo/SP; Brasil
}

\begin{abstract}
In this work, the chemical history of the SMC is described through an observed sample of planetary nebulae (PNe). Age-abundance relations were derived through the $[\alpha$-elements/H] (oxygen, argon, neon and sulphur) ratios estimated from $\mathrm{PNe}$ of different progenitor masses. In particular, oxygen abundances seem do not decrease due to $\mathrm{O}-\mathrm{N}$ cycle operation. (This work was supported by FAPESP)
\end{abstract}

\section{Introduction}

Oxygen is an $\alpha$-element produced mainly by type II supernova events and generally used as archaeological tracer of the chemical evolution of stellar systems. The abundance of oxygen is better determined in planetary nebulae (PNe) than in atmospheres of late-type stars, because $\mathrm{O}$ emission lines are remarkably intense even in metal-poor PNe.

It is usually assumed that if the measured abundances of oxygen in $\mathrm{PNe}$ really reflect, as a rule, the pristine composition of the interstellar medium. However, it is possible that in some cases a significant reduction of $\mathrm{O}$ abundances can occur through ON-cycle. In this work this problem is discussed through an examination of the age- $\alpha /$ element relations for a sample of SMC PNe.

\section{Determination of age-abundance relations}

The abundance data of the $\alpha$-elements $\mathrm{O}, \mathrm{Ne}, \mathrm{S}$ and $\mathrm{Ar}$ are from Costa et al. 2000 and, in some cases, complemented by the data from the literature.

Ages are derived from HR diagrams using the theoretical tracks of Vassiladis \& Wood (1993), as shown in Fig. 1. Effective temperatures $\left(\mathrm{T}_{\text {eff }}\right)$ of $\mathrm{PNe}$ progenitors were estimated from $(\lambda 4686 / \mathrm{H} \beta)$ or $(\lambda 5007) / \mathrm{H} \beta)$ ratios (Kaler \& Jacoby 1989,1991$)$. Progenitor luminosities $\left(\mathrm{L} / \mathrm{L}_{\odot}\right)$ were derived from relations of $\mathrm{V}$ magnitude with $\mathrm{H} \beta$ absolute flux, $\mathrm{T}_{\text {eff }}$, and extinction constant from Kaler \& Jacoby 1989. Bolometric correction are from Kurucz (1979) and Code et al. (1976) and a SMC distance of $57.5 \mathrm{kpc}$ was used to transform $\mathrm{V}$ into $\mathrm{L} / \mathrm{L}_{\odot}$. 

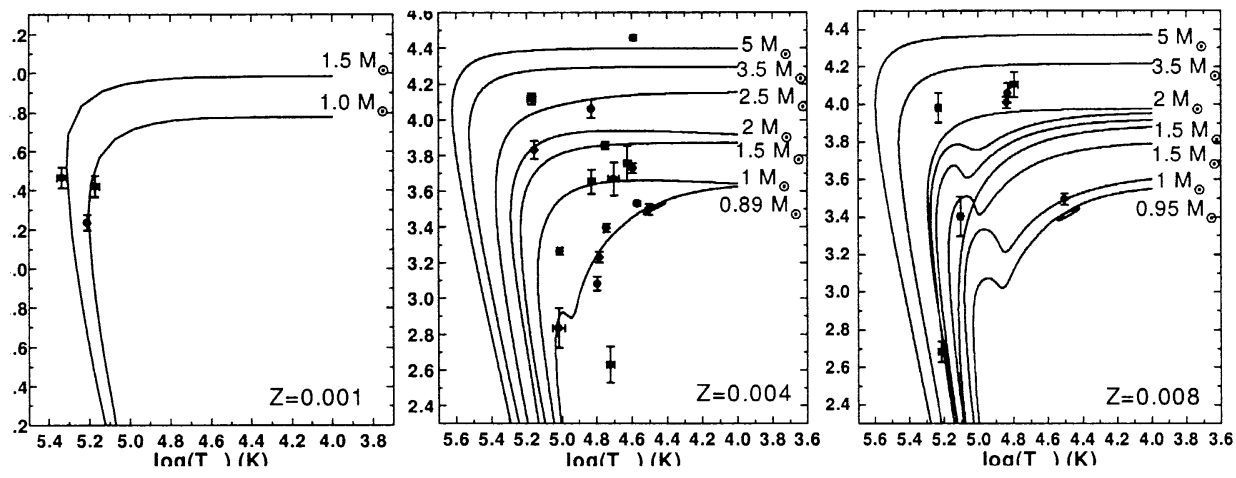

Figure 1. HR diagrams of $\mathrm{PNe}$ progenitors of different metallicities $\mathrm{Z}$ and final stages: $\mathrm{H}, \mathrm{H}$ and $\mathrm{He}$ and He burners.

\section{Analysis and conclusion}

The age-abundance diagrams are displayed in Fig. 2 for $[\mathrm{O} / \mathrm{H}]$ and other $\alpha$ elements. On the average, the linear relations between $[\mathrm{O} / \mathrm{H}]$ and $[\alpha / \mathrm{H}]$ vs. age are the same. This indicates that oxygen abundances can be used as chemical evolution tracers, since they represent the pristine composition of the interstellar medium at the epoch of the formation of the PNe progenitors.
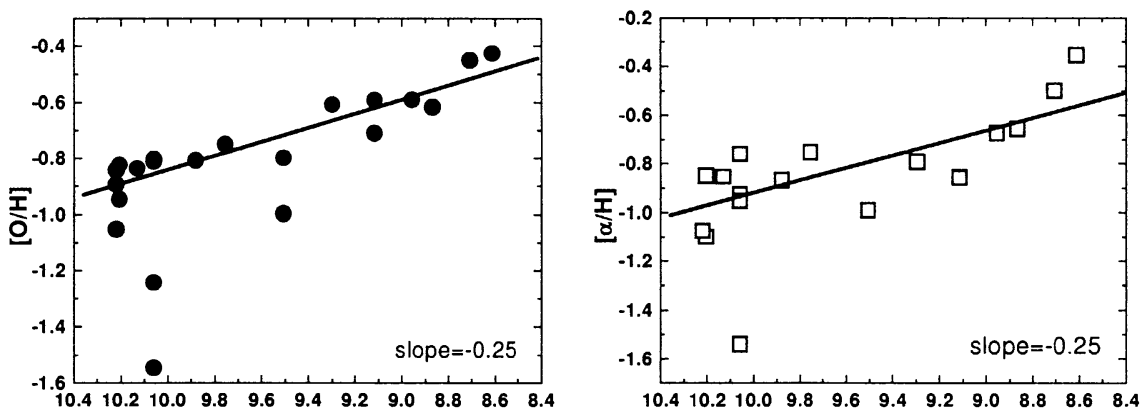

Figure 2. $\alpha=(\mathrm{Ar}+\mathrm{Ne}+\mathrm{S}) / 3$ and $[\mathrm{X} / \mathrm{H}]=\log (\mathrm{X} / \mathrm{H})-\log (\mathrm{X} / \mathrm{H})_{\odot}$.

\section{References}

Code, A.D., Bless, R.C., Davis, J., Brown, R.H. 1976,ApJ, 203, 417

Costa, R.D.D., de Freitas Pacheco, J.A., Idiart, T.P. 2000, A\&AS, 145, 467

Kaler, J.B. \& Jacoby, G.H. 1989,ApJ, 345, 8

Kaler, J.B. \& Jacoby, G.H. 1991,ApJ, 382, 134

Kurucz, R.L. 1979, ApJS, 40, 1

Vassiladis, E. \& Wood, P.R. 1993, ApJ, 413, 641 